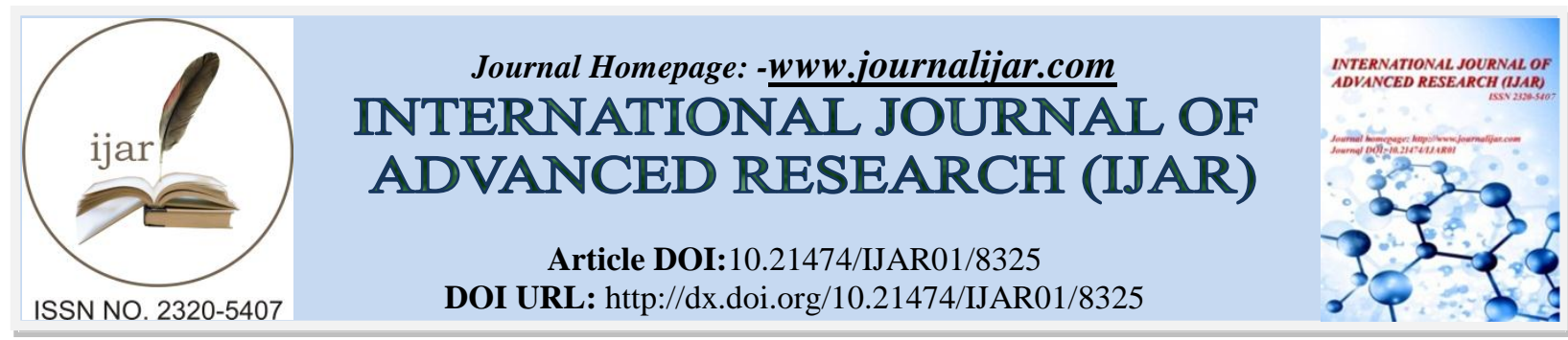

RESEARCH ARTICLE

\title{
NANOTECHNOLOGY IN CIVIL ENGINEERING - APPLICATIONS AND MANAGEMENT.
}

\author{
B. Raj Preethan and D. Rajkumar. \\ Infrastructure Engineering and Management, Department of Civil Engineering ThiagarajarCollegeofEngineering, \\ Madurai- 625015.
}

\section{Manuscript Info}

Manuscript History

Received: 04 November 2018

Final Accepted: 06 December 2018

Published: January 2019

\begin{abstract}
The phenomenal compound and physical properties of Nano materials empower novel applications extending from vitality protection to antimicrobial properties and self-cleaning surfaces to secondary quality improvement in various subjects. The Construction material properties can be enhanced by presenting trademark structures on the nanometer scale. Nanotechnology incorporates the control of the one of a kind properties of issue existing at the "nanoscale". Bunches of iotas and particles show properties very not quite the same as those found at bigger scales. Subsequently, produced nanomaterials (MNMs) and nanocomposites are being considered for different uses in the development and related framework ventures. This order speaks to a chance to increase remarkable understanding into the interesting marvels existing at the nanoscale and to utilize that information to design materials/gadgets with novel attributes. This paper features the advancements in the field of Nanotechnology and specifically to toss light on a portion of the uses of this very developing innovation in Civil Engineering and Design area. Accentuation has been given on the ongoing progressions and the conceivable zones where Nanotechnology and Nano materials may discover its applications in structural designing and related spaces.
\end{abstract}

Copy Right, IJAR, 2017,. All rights reserved.

\section{Introduction:-}

Nanotechnology can be characterized as:-

1. The capacity to make materials, gadgets and frameworks, through control of subject matter in the nanoscale.

2. The process of capitalizing the properties and phenomena happening at the nanoscale.

Nanotechnology is an umbrella term covering an extensive variety of advances concerned about structures and procedures on the nanometer scale. A nanometer is one-billionth of a meter $(10-9 \mathrm{~m})$, and marks an edge where quantum physical impacts progressively assume a vital job. In view of its capability to change generally entire fields of innovation, nanotechnology is viewed as a key innovation which won't just impact mechanical advancement sooner rather than later, however will likewise have definitive financial, biological and social implications. There isno general recognition for the perceived meaning of nanotechnology till date. In an both practical and theoretical minded methodology the present report utilizes the accompanying definition which states as the following:-

Corresponding Author:-B. Raj Preethan.

Address:-Infrastructure Engineering and Management, Department of Civil Engineering ThiagarajarCollegeofEngineering, Madurai- 625015. 
1. Nanotechnology manages structures, which are smaller than $100 \mathrm{~nm}$ in either one dimension.

2. Nanotechnology adventures trademark impacts and wonders, which happen in the transitional zone between the atomic and mesoscopic level.

3. Nanotechnology depicts conscious make or potentially control of individual nanostructures.

Important material properties such as hardness, wear and tear resistance and so on can be deliberately enhanced by presenting trademark structures on the nanometer scale. Numerous utilizations of Nano-crystalline materials got from the conveyance of nanoparticles in an earthenware, metallic or polymer matrix. For instance, presenting nanoscale particles in metals enhances the mechanical properties, which can contribute generously to lightweight construction. Polymers altered with nanoparticles have attributes, which lie between those of natural polymers and inorganic ceramics. Conceivable employments of such enhanced materials lie in especially requesting zones of lightweight development or high-temperature applications, yet in addition in mass applications like plastic casings or cladding. A vital present case is the flexible behavior of nanostructured ceramics, which were previously referred as just brittle materials. In practice, this yields an extensive variety of developments along with fired innovation can produce significant upgrades in properties and additional conceivable development in materials with Nano-added substances. The possible area of developments are high-performance concretes with more prominent compressive quality and enhanced wear and tear accompanied with erosion resistance.

There is abundance of conceivable applications for nanotechnology improvements in construction field. For instance, silicon dioxide nanoparticles can be utilized in manufactured silicic acid (Nano silica) as an additive for sprayed and high performance concrete, which enhances bond rigidity and bond shear quality among concrete and reinforcing steel. Nanometer-thin multilayer covering from conducting polymers can make conceivable enhanced insurance against erosion when utilizing carbon steel or stainless steel as a construction material. Other applications can be found in the field of thermal protection for structures by the utilization of windows for transparent thermal protection by applying an invisiblesilver coating of a few nanometers thickness. In exterior structures such as façade design, enhancing self-cleaning, anti-graffiti protection or high scratch and wear resistance in plastics through proper coatings. In the interior, utilization of titanium dioxide nanoparticles as added substances in coatings to shield from staining under artificial and natural light.

Essential structures of nanotechnology are:-

1. Point like structures smaller than $100 \mathrm{~nm}$ in each of the three measurements (e.g. nanocrystals, clusters or molecules)

2. Linear structures which are Nano sized in two measurements (e.g. nanowires, nanotubes and Nano-grooves),

3. Layered structures which are Nano sized in just a single measurement.

4. "Inverse" nanostructures (i.e. pores) and complex structures, for example, supramolecular units or dendrimers.

Without procedures and instruments equipped for assembling and breaking down the essential structures referred to the above, nanotechnology would be incomprehensible.

\section{Cement Hydration Kinetics:-}

Cement is a permeable material characterized by air voids extending in size to nanometer-scale pores created by the water-cement chemical reaction. "Since these nanoscale pores control the properties of the calcium-silicate hydration process, which is the primary bond that holds concrete together, and thus we can say concrete is someway related to a nanoscale material. It is very much essential to have an exact model to represent the rate of response of concrete with water as an element of temperature, water/cement ratio, and grain size, yet this essential data has been exceptionally hard to get utilizing ordinary logical strategies in light of the fact that the responses occur in the nanoscale pores of the concrete gel. Utilization of atomic reverberation response analysis to think about bond hydration at a nanoscale level results in a superior thought of what happens on the surface of the concrete molecule as it hydrates, prompting enhanced industry measures and rules for blending and curing concrete.

\section{Procedure:-}

This strategy uses a beam of nitrogen atoms to probe a reaction. 


\section{Hydration process:-}

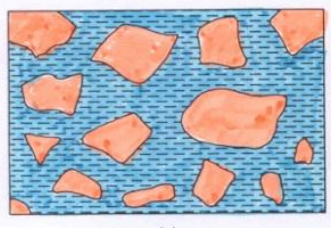

(a)

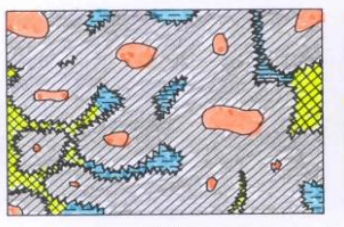

(c)

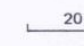

$20 \mu \mathrm{m}$

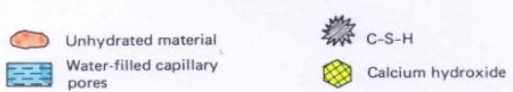

pores

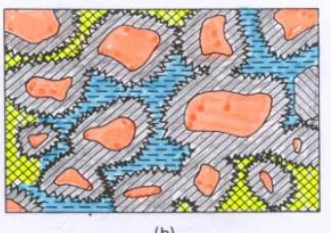

(b)
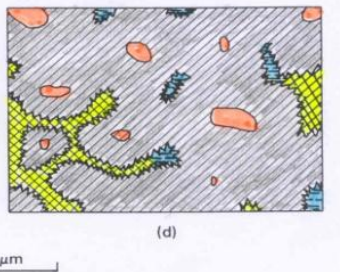

\section{C-S-H gel:-}

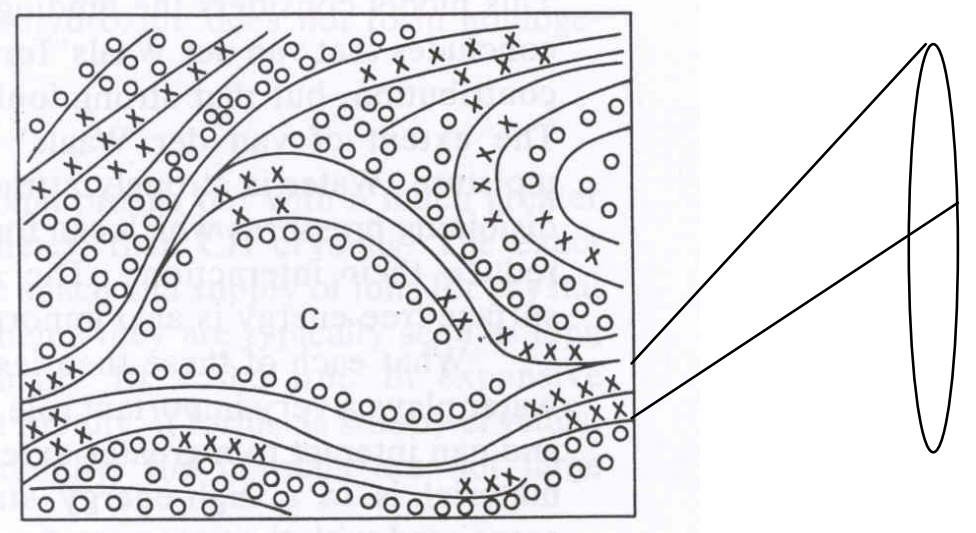

$\mathrm{x}$ - Inter-laminar water

$\mathrm{O}$ - Adsorbed water

$\mathrm{C}$ - Capillary pore - C-S-H layers

g cement grain to locate hydrogen atoms, a necessary component of water, or its reaction products. The results of the probe are plotted in a graph called a hydrogen depth profile, which shows the rate of penetration of the water. This also indicates the arrangement of the various surface layers formed during the reaction.

1. The 20-nanometer-thick surface layer goes about as a semi-porous obstruction that enables water to enter the concrete grain and calcium ions to leach out. But the bigger silicate particles in the concrete are trapped behind this layer.

2. As the reaction proceeds with, a silicate gel layer forms underneath the surface layer, causing swelling inside the cement grain and in the long run prompting breakdown of the surface layer. This breakdown discharges the accumulated silicate into the encompassing arrangement of the solution, where it reacts with calcium ions to form a calcium-silicate hydrate gel, which ties the bond grains together and sets the concrete. This lead to a logical discussion that has been continuing for over a century.

3. The advancement of the hydrogen profile demonstrates the time and event of breakdown of the surface layer. This data can be utilized to think about the concrete setting process with respect to time, temperature, science behind cement and associated components, and different variables. Works are done to measure quantitatively as well as qualitatively how cement reacts with water on a nanoscale. We have to more readily realize how to control the timing of cement setting. 


\section{Nanoscale Silica Fumes:-}
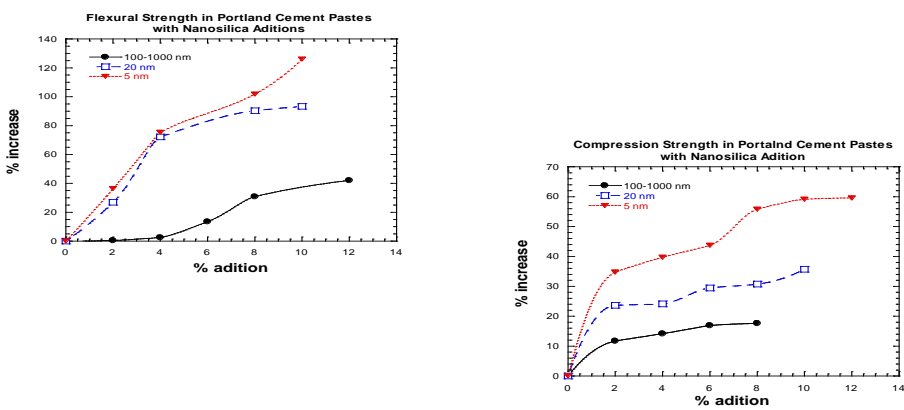

The addition of concrete blends of nanoscale silica fume, an industrial by-product of glass manufacture, is being perceived as a major enhancement in strength and durability of concrete structures exposed to deicing salts.

Silica fume additives works at a nanoscale. We realize it makes for a stronger concrete mix, however in the event that you put excessively in, the solid will end up exhibiting brittleness in in it and becomes weak. We have to all the more likely know how everything cooperates when added together, particularly now that we are including a wide range of new materials, similar to ground granulated blast furnace slag, or superplasticizing synthetic compounds. All can associate in contrasting ways and we have to see quantitative changes that are happening. Henceforth nanotechnology assumes a critical job in monitoring the atomic and sub-atomic level of responses that are going ahead between the different components of concrete with the time associated with every response being included.

\section{Smart Aggregates And Mems:-}

Electromagnetic finite element models are utilized to decide the range and spatial (area) resolution of remote sensors embedded in concrete or soil under different conditions. Robust remote implanted sensors are appropriate for long term field checking of corrosion in rebar, especially for bridge decks. These Smart Aggregates sensors can be installed all through a structure during concrete construction, added appropriate to the mix before placement. The framework is comprised of the Smart Aggregates and a data reader that can be mounted on a vehicle or truck. The reader powers the aggregates as it passes over them and gathers the sensor information onto a PC.

Smart Aggregates contains wireless power receiver and data transmission coils, and is structured utilizing ceramic hybrid integrated circuit technology to withstand mechanical stresses and the high $\mathrm{pH}$ environment of concrete. The aggregates are worked to have a lifetime of more than 50 years. The remote power transmission and information gathering approach wipes out the requirement for and potential issue with batteries, links, and connectors. Model Smart Aggregates have been fabricated and are experiencing dependability estimations.

Entirely, while neither the Cyberliths nor the Smart Aggregate comprise nanotechnology because of their utilization of customary gadgets and their size (a couple of centimeters), they do represent the utilization of implanted sensors and give a vibe for what can be proficient later on as this innovation is decreased in size.

\section{Colloidal Chemistry Of Alkali-Silicate Reaction Gels:-}

Alkali - Silicate Reaction(ASR) happens between alkali from cement and a reactive type of silica from the wrong aggregates, which can result in an alkali/silica gel. If there is a chance that there is sufficient dampness, the gel will grow, harming the concrete adversely. ASR weakens the concrete to the point that it turns out to be extremely vulnerable against external factors, and it's hence that it's been named the AIDS of cement.

The ASR gel expansion mechanism seems to include a stage transformation from amorphous gel to layered structure on the nanoscale. Through the use of neutron scattering and position annihilation spectroscopy, Nano and subnanoscale changes in gel microstructure, which is an element of gel chemistry, temperature and relative humidity can be estimated. 
Utilizing nonreactive aggregates can block ASR in cement. The development of ASR gels by response with various sorts of aggregates can be analyzed utilizing solid-state nuclear magnetic resonance. It can quantify the arrangement of silicate chains on the nanoscale.

\section{Building Steel Through Nanotechnology:-}

Through nanotechnology, Low-carbon, high performance steel(HPS) for bridges can be created. HPS was regarded to require enhanced quality and weldability, and a boost in the general nature of steels utilized in bridges. In 1996 the first of these steels were delivered.

Like black-top asphalt and concrete, steel is a nanostructured material. In the low-carbon HPS steel, copper nanoparticles shape at the steel grain boundaries. The subsequent microstructure changes can make the HPS steel harder, easier to weld, and more corrosion resistant.

An ultra-high strength stainless steel utilizing nanotechnology can likewise be created. The new item, Nanoflex, enables ultra-high strength to be joined with great formability, corrosion resistance and a decent surface wrap up. While as of now being utilized for restorative medical hardware, for example, surgical needles and dental instruments, different areas of utilization can be foreseen, for example, for bridge structural components.

\section{Fly Ash Reactivity Characterization:-}

Through Nanotechnology it is crucial to investigate the communications between fly ash, and the Portland cement gel nanostructure, that influence the quality and strength of concrete. It incorporates the utilization of small angle neutron dispersing to evaluate the progressive changes on a nanoscale as an element of time and fly ash composition and structure.

\section{Delayed Ettringite Formation Damage:-}

Delayed ettringite $\left(\mathrm{Ca}_{6} \mathrm{Al}_{2}\left(\mathrm{SO}_{4}\right)_{3}(\mathrm{OH})_{12} \cdot 26 \mathrm{H}_{2} \mathrm{O}\right)$ is an internal sulphate attack on cement. It tends to be investigated how postponed ettringite forms and causes harm in cement, in changing from an amorphous ettringite gel to nanoscale crystals. It includes the utilization of synchrotron radiation to consider the relationship between ettringite crystal arrangement and concrete extension.

\section{Self-Healing Pavements:-}

The possibility of asphalts or guardrails mending themselves in the wake of being harmed really is the stuff of science fiction. In any case, nanotechnology can be utilized to create self-mending materials made out of particles that can rejoin themselves subsequently after being cut. Work on self-mending polymers as of now is in progress at the University of Illinos where they have built up an auxiliary polymeric material with the capacity to autonomically rejoin the breaks.

Autonomic (unconstrained and spontaneous) rejoining is proficient in this program by consolidating a microencapsulated mending specialist agent and a reactant chemical trigger inside an epoxy grid. A moving toward crack bursts installed microcapsules, discharging mending specialist agent into the break plane through capillary action. Polymerization of the mending specialist agent is activated by contact with the embedded catalyst, holding the break faces.

A comparable procedure has been portrayed in which microsized hollow strands/fibers loaded up with break sealant would be brought into concrete. On the event if the concrete cracks, the fibers would likewise break and discharge sealant. This would be particularly appropriate for bridge piers and column sections experiencing micro-cracking very often and requiring costly epoxy infusion.

Another astonishing property is oneself self-healing property of UHPCs [ultrahigh-performance concretes]. The high fraction of anhydrous [lacking water] material left after the reaction with the water utilized in the underlying blend is a repository for further hydration. At the point when a microcrack occurs, fresh anhydrous surfaces are uncovered. If the example is soaked in water, hydration begins again on these split surfaces. The newly formed hydrates quickly fill the cracks and seal it. 


\section{Concrete Cleanup:-}

Nanotechnology can prompt signs that will have the capacity to shed water. Existing coatings will in general collect grime, which decreases visibility and corrupts the materials after some time. We can make plastic layers that have a nanoscale of roughness that will repel water and dirt, modeled after the coating of the lotus leaf. The lotus leaf, or water lily leaf, displays an exceptional capacity to keep itself perfect clean and dry. Presently nanotechnology can be utilized to replicate the lotus leaf surface and make new items that beat existing no-stick items.

Ordinarily, on a hydrophobic (water-repellent) simple clean surface, particles of dirt are simply moved around by moving water. Be that as it may, on a Lotus Effect surface, dirt and grime is gathered by water drops and washes off.

As of now just a single commercial item is accessible which uses this impact, is exterior house paint. In any case, it is just a short time until the point that the nanotechnology challenges are resolved so this innovation can be conveyed to the market for use with traffic signs and, specifically, traffic activity control gadgets, which require labor intensive, intermittent washing to expel street grime and improve visibility.

Self-cleaning concrete that shields structures from turning dark from toxins in the climate and atmosphere can be created by including particles of the white color titanium dioxide as one of the cement compound.

At the point when titanium dioxide assimilates ultraviolet light, it turns out to be profoundly receptive and separates poisons that come into contact with the solid's surface. The reactive material can eliminate microorganisms and growths and furthermore separate toxins, for example, nitric oxide, sulfur dioxide, and numerous volatile organic aggravates that add to concrete`s darkening.

The Self-Cleaning concrete has just been utilized in a few new structures, incorporating a cutting edge church in Rome called the Dives in Misericordia. The material has applications past keeping concrete surfaces brilliant. Cassar's group has discovered that the concrete can in reality clean the air. Thus this type of concrete may likewise subside natural contamination and outlay the ill effects of environmental pollution.

\section{Conclusion:-}

The quest for enhanced concrete materials proceeds and has no ending. How much the world embraces these new kinds of cement concrete mixes relies upon various elements, including whether the materials address specialized issues, technical needs, the amount they cost, and whether big-time planners, designers, architects and engineers receive them.

The endeavors to bring cement higher than ever of capacity, structure and shape, in any case, is relatively sure to change the customary impression of concrete as a cool, dull, low-tech material. Its utilization is probably going to stretch out as far into the future as it ventures into the past.

1. Nanotechnology is advancing quicker than anticipated.

2. Due to its exceptional qualities notable coordinated effort between players is very much important.

3. It is important to take a shot at the edge of Science - Technology - Market relations.

4. Ultra-High performance materials and parts are one of the ways, not the unique, for a change towards the generation of end user added value.

5. Nanotechnology and its applications are being uncovered as a fundamental device for the ultra-high performance materials development.

\section{References:-}

1. Jaesang lee, Shaily Mahendra and Pedro j. J. Alvarez., "Nanomaterials in the construction industry: a review of their applications and environmental health and safety considerations", 2010

2. B. B. Das and Arkadeep Mitra., "Nanomaterials for construction engineering-a review",2014

3. Radu Olar., "Nanomaterials and Nanotechnologies for Civil Engineering", 2011

4. M. Mahdavinejad et al., "Commercialization strategies for industrial applications of nanomaterials in building construction", advanced materials research, vol. 829, pp. 879-883, 2014

5. P. Ahadi, "Applications of Nanomaterials in construction with an approach to energy issue", advanced materials research, vols. 261-263, pp. 509-514, 2011

6. M. Mahdavinejad et al., "Challenges regarding to usage of nanostructured materials in contemporary building construction", advanced materials research, vol. 829, pp. 426-430, 2014 
7. M. Nazari et al., "Protection of high-tech buildings facades and envelopes with one sided nano-coatings", advanced materials research, vol. 829, pp. 857-861, 2014

8. T.p. Kasharina, "Improving the reliability of shell structures made of composite nanomaterials", solid state phenomena, vol. 265, pp. 365-368, 2017

9. S. Naganathan et al., "Nanotechnology in civil engineering - a review", advanced materials research, vol. 935, pp. 151-154, 2014

10. H. Nabizadeh Rafsanjani and M. Kadivar, "Application of nanotechnology in civil engineering", advanced materials research, vols. 261-263, pp. 520-523, 2011. 